\title{
Safety of biologics in rheumatoid arthritis: data from randomized controlled trials and registries
}

\author{
This article was published in the following Dove Press journal: \\ Biologics:Targets and Therapy \\ 27 January 2015 \\ Number of times this article has been viewed
}

\author{
Catalin Codreanu' \\ Nemanja Damjanov ${ }^{2}$ \\ 'Rheumatology Department, Center \\ of Rheumatic Diseases, Bucharest, \\ Romania; ${ }^{2}$ nstitute of Rheumatology, \\ School of Medicine, University of \\ Belgrade, Belgrade, Serbia
}

\begin{abstract}
Over the past decade, the use of biologics has significantly changed the management of rheumatoid arthritis (RA). Biologics selectively target components of the immune system, resulting in better disease control. However, the growing use of biologics in RA has increased safety concerns among rheumatologists. Randomized controlled trials (RCTs) and registries are the most reliable sources of clinical safety data. Although safety data from RCTs provide certain insights into the clinical safety profile of an agent, strict constraints in study design (eg, exclusion criteria and restrictive treatment protocols) often do not accurately reflect possible safety issues in the use of the agent, either in the clinical setting or over long-term treatment. Registries, on the other hand, are not restrictive regarding patient enrollment, making them more reliable in evaluating long-term safety. A number of registries have been established globally: in Europe, the United States, and Asia. However, the availability of registry data from Eastern Europe is lacking. The notable exceptions so far are registries from the Czech Republic (ATTRA, a registry of patients treated with anti-tumor necrosis factor-alpha drugs) and Serbia (National registry of patients with rheumatoid arthritis in Serbia [NARRAS]). The current report provides an overview of safety data with biologics in RA from RCTs and registries. Availability of regional safety data from Eastern Europe is of great importance to its clinicians for making evidence-based treatment decisions in RA.
\end{abstract}

Keywords: biologic therapy, biologic drugs, adverse events, infections, pregnancy, malignancies

\section{Introduction}

Rheumatoid arthritis (RA) is a disorder characterized by joint and systemic inflammation, joint pain, deformity, and destruction. ${ }^{1}$ Early diagnosis and treatment of RA is essential for the prevention of progressive joint damage. The use of biologics has significantly improved outcomes in patients with RA, making disease remission an attainable goal. ${ }^{2,3}$ The biologics approved for treatment of RA include (in alphabetical order): abatacept, adalimumab, anakinra, certolizumab pegol, etanercept, golimumab, infliximab, rituximab, and tocilizumab. ${ }^{3}$

As the number of patients treated with biologics increases globally, it is crucial to monitor the long-term safety of these agents. The sources of clinical safety data for biologics include randomized controlled trials (RCTs) and registries. Despite being essential in demonstrating the efficacy of drugs and identifying their adverse events (AEs), the validity of results from RCTs is limited by the small number of patients included in the studies, as well as by their short duration. ${ }^{4-6}$ Some AEs are rare and occur only during long-term use of biologic therapy.

Registries, on the other hand, are the most reliable source of long-term safety data. A number of RA registries have been established in Europe, the United States,
Correspondence: Catalin Codreanu Rheumatology Department, Center of Rheumatic Diseases, 5 Thomas Masaryk St, Sector 2, Bucharest, 020983, Romania Tel +40 722204389

Fax +40 21 31। I880

Email ccodreanu@clicknet.ro
Biologics: Targets and Therapy 2015:9 I-6 I

(c) (i) (8) 2015 Codreanu and Damjanov. This work is published by Dove Medical Press Limited, and licensed under Creative Commons Attribution - Non Commercial (unported, v3.0) BY NC License. The full terms of the License are available at http://creativecommons.org/licenses/by-nc/3.0/. Non-commercial uses of the work are permitted without any further
permission from Dove Medical Press Limited, provided the work is properly attributed. Permissions beyond the scope of the License are administered by Dove Medical Press Limited. Information on
how to request permission may be found at: http://www.dovepress.com/permissions.php how to request permission may be found at: http://www.dovepress.com/permissions.php 
and Asia. ${ }^{7}$ Eastern Europe, however, can currently provide data from only two established RA registries: the registry of patients treated with anti-tumor necrosis factor (TNF)-alpha drugs in the Czech Republic (ATTRA) and the National registry of patients with rheumatoid arthritis in Serbia (NARRAS). Consequently, most Eastern European clinicians must extrapolate safety and efficacy data regarding the use of biologics from registries established in other regions. This is of particular concern, since the incidence of tuberculosis (TB) and the risk for reactivation, an AE associated with treatment with TNF inhibitors, is greater in Eastern Europe than in Western Europe. ${ }^{8,9}$ Therefore, more RA registries need to be established in Eastern Europe. This report provides an overview of RA safety data from RCTs (using Cochrane Reviews) and selected registries. Moreover, it highlights the importance of long-term safety data in evidence-based treatment decisions.

\section{RCTs}

The Cochrane Database of Systematic Reviews includes data on biologics used to treat RA and other inflammatory diseases. Data from 160 RCTs (48,676 patients) and 46 open-label extension (OLE) studies (11,954 patients) were used to assess the safety profile of biologics in patients with any disease or medical condition except human immunodeficiency virus. The data were combined across diseases, as it had been assumed that the safety profiles of each biologic would be similar regardless of disease type. The median duration of the RCTs was 6 months, whereas the median duration of the OLE studies was 13 months, allowing longer follow-up. The following nine biologics were compared with placebo: TNF inhibitors (adalimumab, certolizumab pegol, etanercept, golimumab, and infliximab); interleukin-1 receptor antagonist (anakinra); interleukin-6 receptor antagonist (tocilizumab); selective costimulation modulator of T-cells (abatacept); and anti-B-cell (rituximab) therapies. ${ }^{10}$ The odds ratio (OR) was used as a measure of the association between the biologics used and their safety. Event rates less than 10\% were interpreted as an estimate of the risk OR. ${ }^{10}$

The primary safety outcomes were the number of AEs, withdrawals due to AEs, number of serious AEs (SAEs), number of serious infections, diagnosis or reactivation of $\mathrm{TB}$, diagnosis of leukemia or lymphoma, and diagnosis of congestive heart failure (CHF).

\section{Number of AEs}

The number of AEs was defined as the total number of AEs that occurred during the treatment with a particular biologic.
Overall, biologics were associated with more AEs than placebo. Infliximab was associated with a statistically significantly higher number of $\mathrm{AEs}(\mathrm{OR}=1.55 ; 95 \%$ confidence interval [CI]: 1.01-2.35) compared with placebo. However, the numbers of AEs for the other eight biologics were not statistically different from those observed in the placebo groups. The data from the OLE studies were consistent with the RCT results. ${ }^{10}$ The Cochrane Review does not include information on drug survival rates from RCTs.

\section{Withdrawals due to AEs}

Among the nine biologics studied in the RCTs, infliximab was associated with a statistically significantly higher risk of withdrawal (OR $=2.43$; 95\% CI: 1.40-4.14) compared with placebo. With other biologics, the number of withdrawals due to AEs was not statistically different from placebo. Withdrawal rates due to AEs from the OLE studies ranged from $3.7 \%$ (rituximab) to $22.4 \%$ (tocilizumab). ${ }^{10}$

\section{Number of SAEs}

The number of SAEs observed during treatment with any of the nine biologics was not significantly different than the number of SAEs observed during treatment with placebo. However, abatacept $(\mathrm{OR}=0.89 ; 95 \% \mathrm{CI}$ : 0.61-1.26) and tocilizumab $(\mathrm{OR}=0.77 ; 95 \% \mathrm{CI}$ : 0.41-1.45) were associated with fewer SAEs compared with placebo. Pairwise comparisons between the biologics showed that certolizumab pegol was associated with a statistically significant increase in the number of SAEs compared with adalimumab (OR $=1.63$; 95\% CI: $1.01-2.62) .{ }^{10}$

\section{Number of serious infections}

Serious infections were defined as infections associated with death, hospitalization, or the use of intravenous antibiotics. Certolizumab pegol demonstrated a statistically significant increase in serious infections compared with placebo $(\mathrm{OR}=4.75 ; 95 \% \mathrm{CI}: 1.52-18.45)$, as did anakinra $(\mathrm{OR}=4.05$; 95\% CI: 1.22-16.84). Although the other seven biologics did not achieve statistical significance compared with placebo, rituximab was associated with the fewest serious infections $(\mathrm{OR}=0.26 ; 95 \% \mathrm{CI}: 0.03-2.16){ }^{10}$

\section{TB diagnosis and reactivation, $\mathrm{CHF}$, and cancer diagnosis}

TB reactivation was diagnosed in 20 of 10,000 individuals treated with biologics, compared with four of 10,000 individuals treated with placebo. A trend toward an increase in reactivation of TB was observed only among patients treated 
with biologics. TB reactivations reported in the OLE studies ranged from $0 \%$ to $0.6 \%$. The small number of TB cases precluded further analysis. Similarly, the number of cases of CHF and lymphoma were insufficient for a full analysis. ${ }^{10}$ Longer follow-up studies are necessary for an accurate assessment of rare SAEs such as TB, cancer, or CHF.

In summary, the following key safety data were obtained from the Cochrane Database of Systemic Reviews:

- biologics were associated with significantly higher rates of total AEs and withdrawals due to AEs in comparison with placebo;

- infliximab was associated with a significantly higher rate of total AEs compared with placebo;

- certolizumab pegol and anakinra were associated with a significantly higher risk of serious infections in comparison with placebo;

- infliximab was associated with a significantly higher risk of withdrawals due to AEs in comparison with placebo; and

- the comparative risk estimates of TB reactivation, lymphoma, and CHF could not be calculated due to the limited data available from RCTs and OLE studies.

\section{Registry data in RA}

In the past decade, several registries for patients with RA have been established worldwide (Table 1).,11-27 Unlike RCTs, registries have neither strict patient exclusion criteria nor restricted treatment protocols. Therefore, they are a useful tool in evaluating long-term safety of biological agents. The registries have provided valuable safety data about biologics, including incidence of TB, discontinuation rates due to AEs, effects on pregnancy, and occurrence of malignancies. ${ }^{28-31}$ Data from the Danish DANBIO registry showed that patients treated with etanercept had the longest drug survival times (76 months), compared with 52 months for adalimumab and 26 months for infliximab based on Kaplan-Meier estimates. ${ }^{18}$

\section{Incidence of TB}

The British Society for Rheumatology Biologics Register (BSRBR) is a nationwide registry for patients with RA treated with biologics. In an analysis of 10,712 patients from the BSRBR treated with a TNF inhibitor, the rate of TB was three- to fourfold higher in patients receiving infliximab or adalimumab than in those receiving etanercept. ${ }^{32}$ These results were confirmed by the Research Axed on Tolerance of Biotherapies registry of France. The data regarding TB cases had been collected for 3 years among French patients
Table I Registries of patients with rheumatoid arthritis

\begin{tabular}{|c|c|c|c|c|}
\hline Country & $\begin{array}{l}\text { Name of } \\
\text { registry }\end{array}$ & $\begin{array}{l}\text { Start } \\
\text { date }\end{array}$ & $\begin{array}{l}\text { Number } \\
\text { of patients } \\
\text { treated with } \\
\text { TNF inhibitors }\end{array}$ & Reference \\
\hline The & DREAM & 2003 & 546 & 7 \\
\hline \multicolumn{5}{|l|}{ Netherlands } \\
\hline Sweden & $\begin{array}{l}\text { ARTIS (STURE, } \\
\text { SSATG) }\end{array}$ & 1999 & 7,354 & 11 \\
\hline UK & BSRBR & 2001 & $\mathrm{II}, 757$ & 12 \\
\hline Germany & RABBIT & 2001 & 5,279 & 13 \\
\hline Spain & BIOBADASER & 2000 & 5,493 & 14,15 \\
\hline Norway & NOR-DMARD & 2000 & 4,683 & 16 \\
\hline Denmark & DANBIO & 2000 & 3,056 & 17,18 \\
\hline Serbia & NARRAS & 2008 & 3,072 & 19,20 \\
\hline Czech & ATTRA & 2001 & $>2,000$ & 21 \\
\hline \multicolumn{5}{|l|}{ Republic } \\
\hline Italy & LORHEN & 1999 & 1,114 & 22 \\
\hline Switzerland & SCQM & 1997 & 2,364 & 23 \\
\hline Greece & HBRT & NA & 715 & 24 \\
\hline Japan & REAL & 2005 & $\mathrm{I}, \mathrm{I} 44$ & 25 \\
\hline USA & CORRONA & 2002 & 8,755 & 26 \\
\hline France & RATIO & 1997 & $|, 57|$ & 27 \\
\hline
\end{tabular}

Notes: Registries are a reliable source of long-term safety data and supplement safety information obtained from randomized controlled trials. ATTRA is a registry of patients treated with anti-TNF-alpha drugs; BIOBADASER is a Spanish registry of biological therapies in rheumatic diseases.

Abbreviations: ARTIS, Anti-rheumatic Therapy in Sweden; ATTRA, Registry of patients treated with anti-tumor necrosis factor (TNF)-alpha drugs in the Czech Republic; BIOBADASER, Spanish registry of biological therapies in rheumatic diseases; BSRBR, British Society for Rheumatology Biologics Register; CORRONA, Consortium of Rheumatology Researchers of North America; DANBIO, Danish registry for biological treatment in rheumatology; DREAM, Dutch RhEumatoid Arthritis Monitoring; HBRT, Helenic Biologic Registry; LORHEN, Lombardy Rheumatology Network; NA, not available; NARRAS, National registry of patients with rheumatoid arthritis in Serbia; NOR-DMARD, Norwegian Disease-Modifying Anti-Rheumatic Drug; RABBIT, Rheumatoid Arthritis Observation of Biologic Therapy; RATIO, Research Axed on Tolerance of Biotherapies; REAL, Registry of Japanese Rheumatoid Arthritis Patients for Long-term Safety; SCQM, Swiss Rheumatoid Arthritis Cohort; SSATG, Southern Sweden Antirheumatic Therapy Group; STURE, Stockholm TNF-alpha follow-up registry; TNF, tumor necrosis factor.

treated with TNF inhibitors for any indication. Annual incidence of TB was 9.3/100,000 patients for individuals treated with etanercept, compared with 187.5/100,000 and 215.0/100,000 patients for individuals treated with infliximab and adalimumab, respectively. ${ }^{33}$ Thus, the data found in either registry demonstrated a lower rate of TB observed in patients treated with etanercept compared with those treated with either infliximab or adalimumab.

\section{Discontinuation rates due to $\mathrm{AEs}$}

The Danish registry for biological treatment in rheumatology (DANBIO), the Spanish registry of biological therapies in rheumatic diseases (BIOBADASER), and the Hong Kong Society of Rheumatology evaluated TNF inhibitor treatment discontinuation rates due to AEs. The data from 
the three registries showed a lower rate of treatment discontinuation due to AEs in patients with RA who were treated with etanercept compared to those who were treated either with adalimumab, or with infliximab. ${ }^{18,34,35}$

However, data from Rheumatoid Arthritis Observation of Biologic Therapy (RABBIT), BSRBR and Consortium of Rheumatology Researchers of North America (CORRONA) registries showed that, regarding the AEs, similar treatment discontinuation rates for different TNF inhibitors has been observed. These results were confirmed by the data recorded in the ATTRA registry of the Czech Republic regarding patients with ankylosing spondylitis. Ankylosing spondylitis is a chronic inflammatory disease of the axial skeleton and peripheral joints. AEs were the most frequent reason for discontinuation of treatment in ATTRA with similar discontinuation rates among TNF inhibitors: $30.4 \%$ of the cases with etanercept treatment, $37.5 \%$ of the cases with adalimumab treatment, and $26.5 \%$ of the cases with infliximab treatment. ${ }^{21}$ These results suggest that treatment discontinuation rates (due to AEs) among TNF inhibitors vary from region to region. However, the combined data from patients with RA and ankylosing spondylitis need to be interpreted with caution as they involve different disease populations.

\section{AEs during pregnancy}

The BSRBR collected data on AEs during pregnancy in patients with RA treated with TNF inhibitors. A total of 130 pregnancies were reported among these patients, with 88 live births. The spontaneous abortion rate increased by $33 \%$ in the patients who had been treated with TNF inhibitors in combination with methotrexate/leflunomide, $24 \%$ in patients treated with TNF inhibitors alone, $17 \%$ among those who had prior exposure to TNF inhibitors, and $10 \%$ in the placebo group. These results suggest that treatment with TNF inhibitors in combination with synthetic disease-modifying antirheumatic drugs (DMARDs) significantly increase the risk of miscarriage compared with treatment with TNF inhibitors alone and that great caution should be taken when treating women planning pregnancy with TNF inhibitors. ${ }^{30}$

\section{Incidence of malignancies}

A meta-analysis was conducted to assess the risks of malignancies associated with TNF inhibitor treatment. Seven registries reported malignancy incidence rates: Anti-rheumatic Therapy in Sweden (ARTIS), the US National Data Bank for Rheumatic Diseases, CORRONA, the US Disease-Modifying Anti-Rheumatic Drug Intervention and Utilization Study (RADIUS), RABBIT, the Spanish registry of biological therapies in rheumatic diseases, and BSRBR. The analysis indicated that treatment with TNF inhibitors was not associated with increased risks of malignancies when compared with treatment with traditional DMARDs $(\mathrm{OR}=0.95 ; 95 \% \mathrm{CI}: 0.85-1.05) .{ }^{29}$ Additionally, the risk of lymphoma in patients with RA was assessed in the Anti-rheumatic Therapy in Sweden registry, the US National Databank for Rheumatic Diseases, and CORRONA registry. The data from the three registries demonstrated that the patients treated with TNF inhibitors did not have an increased risk of lymphoma when compared with the patients treated with DMARDs (OR $=1.1$; 95\% CI: 0.70-1.51). ${ }^{29}$ However, treatment with TNF inhibitors may increase the risk of skin cancer in patients with RA. For example, treatments with infliximab and etanercept were associated with melanoma $(\mathrm{OR}=2.6 ; 95 \% \mathrm{CI}: 1.0-6.7 ; P=0.056$ and $\mathrm{OR}=2.4 ; 95 \%$ CI: $1.0-5.8 ; P=0.054$, respectively) and non-melanoma skin cancers $(\mathrm{OR}=1.7 ; 95 \% \mathrm{CI}: 1.3-2.2 ; P<0.001$ and $\mathrm{OR}=1.2$; 95\% CI: $1.0-1.5 ; P=0.081$, respectively). ${ }^{36}$

In summary, the following long-term safety data were obtained from the registries established in Europe, USA, and Asia:

- the risk of TB was significantly lower with etanercept treatment compared to that with both infliximab and adalimumab treatments;

- treatment with TNF inhibitors did not increase the risk of malignancies, particularly lymphoma;

- treatment with TNF inhibitors may have increased the risk of skin cancers;

- the treatment discontinuation rate due to AEs may be lower with etanercept compared to that with adalimumab or infliximab; and

- pregnant women may need to discontinue treatment with TNF inhibitors.

\section{Discussion}

RCTs and registries are essential sources of clinical safety information for biologic agents. However, RCTs do not accurately reflect the use of biologic agents either long-term or in the clinical setting. Due to serious risks associated with biologics, it is important to obtain proper risk estimates before administering them to patients with RA. Typically, RCTs have restrictive patient exclusion criteria and restrictive treatment protocols, leaving only $30 \%-40 \%$ of patients with RA eligible for inclusion. For example, patients could be excluded from an RCT due to their age, disease-related characteristics, co-treatments, or social background. Registries, on the other hand, include all patients with RA and supply 
long-term data about treatment, thus providing more realistic estimates of potential risks of biologics. ${ }^{4,14}$

The 160 RCTs included in the Cochrane database for biologics were short-term, with a median duration of 6 months. As a result, there were an insufficient number of cases of several outcomes (eg, TB diagnoses/reactivation, lymphoma, and $\mathrm{CHF}$ ) to make a thorough analysis possible. The OLE studies were longer in duration and provided risk estimates of TB reactivation. However, it was not possible to evaluate the impact of biologics on serious malignancies and infections. The registries discussed, however, provided data on treatment discontinuation rates due to AEs, the incidence of TB and cancer, and the effects of biologics on pregnancy. ${ }^{28}$

Therefore, registries are necessary to complete the safety data obtained from RCTs. However, multiple factors may lead to discrepancies when comparing registry safety data from different regions. For example, patients may respond differently to biologics due to their genetic background. ${ }^{37}$ The observed differences in safety may also depend on the comorbidity profiles of patients included in the registries of a specific country. Additionally, background rates of opportunistic infections (eg, TB) in the population may play a role in the discrepancy of safety outcomes reported per region. ${ }^{4}$ For instance, the five countries with the largest number of TB cases in 2009 were India (1.6-2.4 million), People's Republic of China (1.1-1.5 million), South Africa (0.40-0.59 million), Nigeria (0.37-0.55 million), and Indonesia (0.35-0.52 million). ${ }^{38}$ Furthermore, there are demonstrated differences in the incidence of TB between Eastern and Western Europe. ${ }^{8,9}$ Consequently, in order to address variations for a specific country, it is important to establish registries worldwide. Eastern Europe, with a limited number of established registries, should extrapolate currently available registry and RCT data from other regions. Safety characteristics for biologics reported in the registries discussed may not translate directly to Eastern European patients with RA. Availability of registry data from Eastern Europe may help clinicians make informed treatment decisions based on local safety data.

\section{Conclusion}

RCTs and registries provide valuable sources of clinical data for improving treatment. Numerous registries of patients with RA have been globally established to supplement safety information obtained from RCTs. It is important, however, to develop registries in all regions in order to address specific variations in patient profiles for each country. At present, available registry and RCT data could help physicians make informed treatment decisions and improve treatment of patients with RA.

\section{Acknowledgments}

Editorial/medical writing support was provided by S Mallik at ACUMED, and Mukund Nori, PhD, MBA, CMPP, of Engage Scientific Solutions (Southport, CT, USA), provided support for responding to reviewer comments and revisions; both efforts were funded by Pfizer Inc. The authors would also like to thank Zorica Ivkovic of EUTREND Ltd (Belgrade, Serbia) for language editing.

\section{Author contributions}

Both authors made substantial contributions to conception and design, acquisition of data, or analysis and interpretation of data, as well as drafting of the article or revising it critically for important intellectual content. Both authors gave final approval of the version to be published.

\section{Disclosure}

The authors report no conflicts of interest in this work. No compensation was received for authoring this manuscript.

\section{References}

1. Harris ED Jr. Rheumatoid arthritis. Pathophysiology and implications for therapy. N Engl J Med. 1990;322(18):1277-1289.

2. Saleem B, Walsh CA, Emery P. Remission in inflammatory arthritis: a new immunological target. Immunotherapy. 2011;3(4):459-463.

3. van Vollenhoven RF. Treatment of rheumatoid arthritis: state of the art 2009. Nat Rev Rheumatol. 2009;5(10):531-541.

4. Curtis JR, Jain A, Askling J, et al. A comparison of patient characteristics and outcomes in selected European and US rheumatoid arthritis registries. Semin Arthritis Rheum. 2010;40(1):2-14. e1.

5. Geborek P, Crnkic M, Petersson IF, Saxne T; South Swedish Arthritis Treatment Group. Etanercept, infliximab, and leflunomide in established rheumatoid arthritis: clinical experience using a structured follow up programme in southern Sweden. Ann Rheum Dis. 2002;61(9):793-798.

6. Wolfe F, Michaud K, Gefeller O, Choi HK. Predicting mortality in patients with rheumatoid arthritis. Arthritis Rheum. 2003;48(6): 1530-1542.

7. Kievit W, Fransen J, Oerlemans AJ, et al. The efficacy of anti-TNF in rheumatoid arthritis, a comparison between randomised controlled trials and clinical practice. Ann Rheum Dis. 2007;66(11):1473-1478.

8. Global Tuberculosis Report 2014. Geneva: World Health Organization; 2014. Available from: http://www.who.int/tb/publications/global_report/ en/. Accessed September 23, 2014.

9. Migliori GB, Centis R. Problems to control TB in eastern Europe and consequences in low incidence countries. Monaldi Arch Chest Dis. 2002;57(5-6):285-290.

10. Singh JA, Wells GA, Christensen R, et al. Adverse effects of biologics: a network meta-analysis and Cochrane overview. Cochrane Database Syst Rev. 2011(2):CD008794.

11. Askling J, Fored CM, Geborek P, et al. Swedish registers to examine drug safety and clinical issues in RA. Ann Rheum Dis. 2006;65(6): $707-712$.

12. Mercer LK, Galloway JB, Lunt M, et al. The influence of anti-TNF therapy upon incidence of non-melanoma skin cancer (NMSC) in patients with rheumatoid arthritis (RA): results from the BSR Biologics Register (BSRBR) [abstract]. Arthritis Rheum. 2009; 60 Suppl 10:2062. 
13. Strangfeld A, Hierse F, Rau R, et al. Risk of incident or recurrent malignancies among patients with rheumatoid arthritis exposed to biologic therapy in the German biologics register RABBIT. Arthritis Res Ther. 2010;12(1):R5.

14. Gómez-Reino JJ, Carmona L, Valverde VR, Mola EM, Montero MD; BIOBADASER Group. Treatment of rheumatoid arthritis with tumor necrosis factor inhibitors may predispose to significant increase in tuberculosis risk: a multicenter active-surveillance report. Arthritis Rheum. 2003;48(8):2122-2127.

15. Descalzo MÁ, Carmona L; Grupo de Estudio BIOBADASER. [Biobadaser 2.0: analysis and trends in 2009]. Rheumatol Clin. 2010;6(5):240-243. Spanish.

16. Kvien TK, Heiberg MS, Lie E, et al. A Norwegian DMARD register: prescriptions of DMARDs and biological agents to patients with inflammatory rheumatic diseases. Clin Exp Rheumatol. 2005;23(5 Suppl 39): S188-S194.

17. Hetland ML. DANBIO: a nationwide registry of biological therapies in Denmark. Clin Exp Rheumatol. 2005;23(5 Suppl 39):S205-S207.

18. Hetland ML, Christensen IJ, Tarp U, et al; All Departments of Rheumatology in Denmark. Direct comparison of treatment responses, remission rates, and drug adherence in patients with rheumatoid arthritis treated with adalimumab, etanercept, or infliximab: results from eight years of surveillance of clinical practice in the nationwide Danish DANBIO registry. Arthritis Rheum. 2010;62(1):22-32.

19. Damjanov N, Dimic A, Lazarevic M, Stefanovic D, Stankovic A; Rheumatology Association of Serbia. Disease activity and working disability in 1775 patients with rheumatoid arthritis - Data from National Registry of patients with Rheumatoid Arthritis in Serbia. Ann Rheum Dis. 2010;69(Suppl 3):671.

20. Ostojic P, Damjanov N, Dimic A, et al. Improved efficacy of rituximab in rheumatoid arthritis when administered regularly at six-month intervals - data from the Serbian registry (NARASS). Ann Rheum Dis. 2012;71(Suppl 3):670.

21. Pavelka K, Forejtová S, Stolfa J, et al. Anti-TNF therapy of ankylosing spondylitis in clinical practice. Results from the Czech national registry ATTRA. Clin Exp Rheumatol. 2009;27(6):958-963.

22. Marchesoni A, Zaccara E, Gorla R, et al. TNF-alpha antagonist survival rate in a cohort of rheumatoid arthritis patients observed under conditions of standard clinical practice. Ann N Y Acad Sci. 2009;1173: 837-846.

23. Du Pan SM, Dehler S, Ciurea A, Ziswiler HR, Gabay C, Finckh A; Swiss Clinical Quality Management Physicians. Comparison of drug retention rates and causes of drug discontinuation between anti-tumor necrosis factor agents in rheumatoid arthritis. Arthritis Rheum. 2009; 61(5):560-568.

24. Flouri I, Drosos A, Skopouli F, et al. Long term follow-up of RA patients of the Hellenic biologics registry: comparison of first versus second anti-TNFA therapy. Ann Rheum Dis. 2009; 68(Suppl 3):430.

25. Komano Y, Tanaka M, Nanki T, et al; REAL Study Group. Incidence and risk factors for serious infection in patients with rheumatoid arthritis treated with tumor necrosis factor inhibitors: a report from the Registry of Japanese Rheumatoid Arthritis Patients for Longterm Safety. J Rheumatol. 2011;38(7):1258-1264.
26. Kremer J. The CORRONA database. Ann Rheum Dis. 2005;64 Suppl 4: iv37-iv41.

27. Mariette X, Gottenberg JE, Ravaud P, Combe B. Registries in rheumatoid arthritis and autoimmune diseases: data from the French registries. Rheumatology (Oxford). 2011;50(1):222-229.

28. Gómez-Reino JJ, Carmona L, Angel Descalzo M; Biobadaser Group. Risk of tuberculosis in patients treated with tumor necrosis factor antagonists due to incomplete prevention of reactivation of latent infection. Arthritis Rheum. 2007;57(5):756-761.

29. Mariette X, Matucci-Cerinic M, Pavelka K, et al. Malignancies associated with tumour necrosis factor inhibitors in registries and prospective observational studies: a systematic review and meta-analysis. Ann Rheum Dis. 2011;70(11):1895-1904.

30. Verstappen SM, King Y, Watson KD, Symmons DP, Hyrich KL; BSRBR Control Centre Consortiu, BSR Biologics Register. AntiTNF therapies and pregnancy: outcome of 130 pregnancies in the British Society for Rheumatology Biologics Register. Ann Rheum Dis. 2011;70(5):823-826.

31. Strangfeld A, Zink A. [Safety of biologic therapy - results from the German biologics register RABBIT]. Dtsch Med Wochenschr. 2014;139(37):1817-1820. German.

32. Dixon WG, Hyrich KL, Watson KD, Lunt M, Galloway J, Ustianowski A; BSRBR Control Centre Consortium, Symmons DP; BSR Biologics Register. Drug-specific risk of tuberculosis in patients with rheumatoid arthritis treated with anti-TNF therapy: results from the British Society for Rheumatology Biologics Register (BSRBR). Ann Rheum Dis. 2010;69(3):522-528.

33. Tubach F, Salmon D, Ravaud P, et al; Research Axed on Tolerance of Biotherapies Group. Risk of tuberculosis is higher with anti-tumor necrosis factor monoclonal antibody therapy than with soluble tumor necrosis factor receptor therapy: the three-year prospective French Research Axed on Tolerance of Biotherapies registry. Arthritis Rheum. 2009;60(7):1884-1894.

34. Gómez-Reino JJ, Carmona L; BIOBADASER Group. Switching TNF antagonists in patients with chronic arthritis: an observational study of 488 patients over a four-year period. Arthritis Res Ther. 2006; 8(1):R29.

35. Mok CC, Kwan C, Chan KY, Lee KL, Tam LS; Hong Kong Society of Rheumatology. Retention rate of the anti-TNF biologics in the treatment of rheumatic diseases and predictive factors for drug withdrawal: data from the Hong Kong Biologics Registry. Ann Rheum Dis. 2012; 71(Suppl 3):663.

36. Wolfe F, Michaud K. Biologic treatment of rheumatoid arthritis and the risk of malignancy: analyses from a large US observational study. Arthritis Rheum. 2007;56(9):2886-2895.

37. Bansard C, Lequerré T, Daveau M, et al. Can rheumatoid arthritis responsiveness to methotrexate and biologics be predicted? Rheumatology (Oxford). 2009;48(9):1021-1028.

38. Global Tuberculosis Report 2013. Geneva: World Health Organization; 2013. Available from: http://apps.who.int/iris/bitstream/10665/91355/1/9789241564656_eng.pdf. Accessed March 10, 2014
Biologics: Targets \& Therapy

\section{Publish your work in this journal}

Biologics: Targets \& Therapy is an international, peer-reviewed journal focusing on the patho-physiological rationale for and clinical application of Biologic agents in the management of autoimmune diseases, cancers or other pathologies where a molecular target can be identified. This journal is indexed on PubMed Central, CAS, EMBase, Scopus

\section{Dovepress}

and the Elsevier Bibliographic databases. The manuscript management system is completely online and includes a very quick and fair peerreview system, which is all easy to use. Visit http://www.dovepress. com/testimonials.php to read real quotes from published authors. 